\title{
Reproducibility of Standing Posture for X-Ray Radiography: A Feasibility Study of the BalancAid with Healthy Young Subjects
}

\author{
Dyah Ekashanti Octorina Dewi, ${ }^{1}$ Albert G. Veldhuizen, ${ }^{2}$ Johannes G. M. Burgerhof, ${ }^{3}$ \\ I Ketut Eddy Purnama, ${ }^{4}$ Peter M. A. van Ooijen, ${ }^{5}$ Michael H. F. Wilkinson, ${ }^{6}$ \\ Tati Latifah Erawati Rajab Mengko, ${ }^{7}$ and Gijsbertus Jacob Verkerke ${ }^{1,8}$
}

\begin{abstract}
${ }^{1}$ Department of BioMedical Engineering, University Medical Center Groningen, University of Groningen, Antonius Deusinglaan 1, 9713 AV Groningen, The Netherlands; ${ }^{2}$ Department of Orthopedic Surgery, University Medical Center Groningen, University of Groningen, Hanzeplein 1,9713 GZ Groningen, The Netherlands; ${ }^{3}$ Department of Epidemiology and Statistics, University Medical Center Groningen, University of Groningen, Hanzeplein 1, 9713 GZ Groningen, The Netherlands;

${ }^{4}$ Department of Electrical Engineering, Institut Teknologi Sepuluh Nopember, Campus ITS Keputih, Surabaya 60111, Indonesia; ${ }^{5}$ Department of Radiology, University Medical Center Groningen, University of Groningen, Hanzeplein 1, 9713 GZ Groningen, The Netherlands; ${ }^{6}$ Johann Bernoulli Institute of Mathematics and Computer Science, University of Groningen, Nijenborgh 9, 9747 AG Groningen, The Netherlands; ${ }^{7}$ Biomedical Engineering Research Division, School of Electrical Engineering and Informatics, Institut Teknologi Bandung, Ganesha 10, Bandung 40132, Indonesia; and ${ }^{8}$ Department of Biomechanical Engineering, University of Twente, Enschede, The Netherlands
\end{abstract}

(Received 22 December 2009; accepted 3 May 2010; published online 15 May 2010)

Associate Editor Kyriacos A. Athanasiou oversaw the review of this article.

\begin{abstract}
Unreliable spinal X-ray radiography measurement due to standing postural variability can be minimized by using positional supports. In this study, we introduce a balancing device, named BalancAid, to position the patients in a reproducible position during spinal X-ray radiography. This study aimed to investigate the performance of healthy young subjects' standing posture on the BalancAid compared to standing on the ground mimicking the standard X-rays posture in producing a reproducible posture for the spinal X-ray radiography. A study on the posture reproducibility measurement was performed by taking photographs of 20 healthy young subjects with good balance control standing on the BalancAid and the ground repeatedly within two consecutive days. We analyzed nine posterior-anterior (PA) and three lateral (LA) angles between lines through body marks placed in the positions of T3, T7, T12, L4 of the spine to confirm any translocations and movements between the first and second day measurements. No body marks repositioning was performed to avoid any error. Lin's CCC test on all angles comparing both standing postures demonstrated that seven out of nine angles in PA view, and two out of three angles in LA view gave better reproducibility for standing on the BalancAid compared to standing on the ground. The PA angles concordance is on average better than that of the LA angles.
\end{abstract}

Address correspondence to Gijsbertus Jacob Verkerke, Department of BioMedical Engineering, University Medical Center Groningen, University of Groningen, Antonius Deusinglaan 1, 9713 AV Groningen, The Netherlands. Electronic mail: d.e.o.dewi@med. umcg.nl, a.g.veldhuizen@orth.umcg.nl, j.g.m.burgerhof@epi.umcg.nl, ketut@ee.its.ac.id, p.m.a.van.ooyen@rad.umcg.nl, m.h.f.wilkinson@ rug.nl, tmengko@itb.ac.id, g.j.verkerke@med.umcg.nl
Keywords-Posture reproducibility, Spinal X-ray radiography, Balancing device.

\section{INTRODUCTION}

For the reason that X-ray radiography is an economic, user-friendly, and readily available tool to perform direct visualization of the anatomy of interest to many clinical questions, the Scoliosis Research Society (SRS) has endorsed the full spinal X-ray radiographs as a standardized procedure for assessing the scoliosis progression over time taken in standing anterior-posterior (AP) and lateral (LA) views. ${ }^{7,8,12,22,29}$ Although the $\mathrm{X}$-ray radiograph measurement is effective, $\mathrm{X}$-ray radiography has detrimental radiation effect. ${ }^{5,25,26}$ In view of the above reason, the risk of having unnecessary additional X-rays and inaccurate measurement of scoliosis progression due to erroneous variability in the production of a spinal X-ray radiograph should be minimized. The studies of Capasso et al., ${ }^{5}$ Oda et al., ${ }^{25}$ and Pruijs et al $^{26}$ reported that most inaccuracies in the curvature evaluation are mainly influenced by inparticular postural variabilities of the subject and equipment positional changes during X-ray radiography. In accordance with the abovementioned studies, other studies $^{8,17,28}$ also confirmed that errors due to patient posture cause differences in determination of the curvature. In addition, Beauchamp ${ }^{2}$ found that diurnal variation had statistically and clinically significant 
changes in the curvature measurement for moderate to severe curves. In this regard, postural variability has evidently hampers the production of accurate and reproducible spinal $\mathrm{X}$-ray radiographs.

The arm position is also critical for a revealing $\mathrm{X}$-ray radiograph. Seeing that the global sagittal balance is considerably influenced by the arm position during standing upright, it is necessary to establish an accurate arm position in the LA view of spinal X-ray radiography which generates the least negative sagittal vertical axis and performs the smallest variability. ${ }^{1,9,11,19}$ However, the arm position triggers more complexity in the spinal X-ray radiograph as the arms and the glenohumeral joint shadows block the visibility of the vertebral landmarks in the LA view. ${ }^{1,9,11,19}$ Previous investigations have also found that the arm position in the spinal X-ray does not only influence the visibility of the vertebrae in the LA-position and affect the sagittal spinal alignment in the analysis of spinal deformity, but this arm position also plays an important role in the generation of a reliable three-dimensional representation of the spine and in the correction of the spinal disorder. ${ }^{10,27,30}$ In addition to demonstrating the importance of having a proper arm position, such studies also presented several armpositioning strategies, which may produce an optimal vertebral visibility and global sagittal balance. The best method which most studies have recommended is generally to position the arms on the clavicular position with elbows flexed and fists resting on ipsilateral clavicles. ${ }^{1}$ This method provides a good representation of a functional balance while still allowing an adequate LA radiographic visualization of the spine.

Nevertheless, in addition to postural reproducibility and arm-positioning factors, a reliable spinal X-ray is also affected by the balance capability of the subject. Indeed, preceding studies s, $^{3,24}$ found that scoliotic patients with progressive curvature are likely to have postural equilibrial dysfunction. Moreover, a strong correlation between the standing imbalances in scoliotic patients and the stability of specific postural alterations have also been demonstrated in the study of Le Blanc et al. ${ }^{15}$ and Nault et al..$^{23}$ This can be indicative that the posture imbalances due to severe scoliosis bring about inaccuracy of the progression measurement of the spinal X-ray radiograph over time.

In view of the high impact of the reproducible posture, arm positioning, and balance capability in yielding reliable measurements, a practical recommendation for patient positioning during X-ray radiography has already been issued by the SRS. ${ }^{29}$ The recommendation, which recognizes the vertebral body line and axis system and takes the balance posture into account, may help to define the nature of the global spinal deformities with X-ray measurement. Further- more, to align the subject's global axis system with the film plane, the SRS has advised to use supports to position the anterior superior iliac spine parallel to the film plane. Such position supports seem to be useful to solve the alignment problem, nevertheless, have not accommodated the postural balance of the subject. Therefore, it is important to take the balance control into account to minimize any inaccuracy or irreproducibility in the spinal X-ray analysis.

In this study, we address the improvement of the standing posture reproducibility during X-ray radiography by means of a balancing plate, BalancAid, as illustrated in Fig. 1. We propose a minimized erroneous variability in the reproducibility of the spinal $\mathrm{X}$-ray radiography and a more accurate Cobb angle determination leading to better diagnosis. The BalancAid consists of a square board with a cylindrical disk in the center point attached at the center of the bottom. Such design realizes a forced balance not only in the sagittal plane, but also in the frontal plane, with the intention that the balance is obtained from all directions. The balancing effect from the device forces the subject to stand balanced and directs the posture in a specific upright position. In addition, using this balancing plate, a well-described arm position can be performed without any supporting bars. We evaluate

(a)

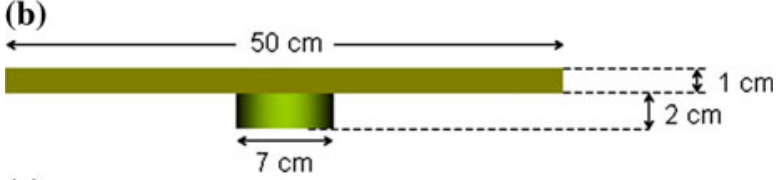

(c)

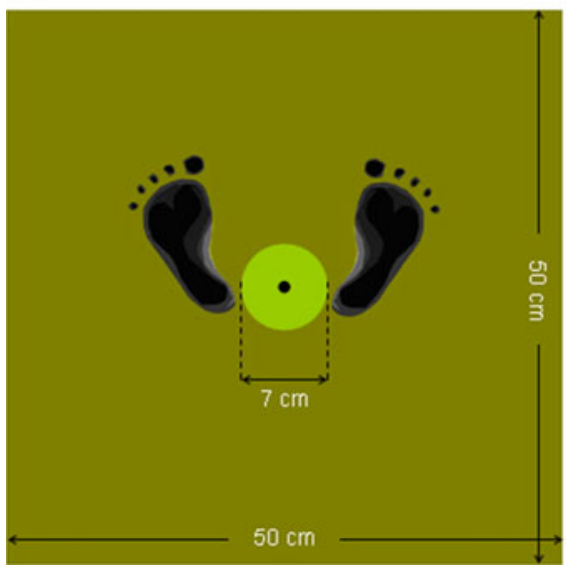

FIGURE 1. The prototype of the BalancAid. (a) Threedimensional view of the BalancAid. (b) Front view of the BalancAid. (c) Bottom view of the BalancAid. This balancing plate consists of the upper rectangular plate and the lower cylindric plate which is attached in the middle bottom of the upper rectangular plate. The footprint is provided from the midline to guide the subject to stand in a neutral standardized stance (sub-talar joint neutral). 
the performance of the BalancAid in improving the posture reproducibility when performing the spinal $\mathrm{X}$-ray radiographs by comparing it to the standing posture on the ground using a conventional procedure. In this regard, our study on the Balanc Aid is performed as a representative of healthy young subjects with good balance control. Our hypothesis is that standing on the BalancAid will improve the posture reproducibility of young patients with minor scoliosis and good balance control, thus is feasible to apply in the monitoring of at least the progression of minor spinal deformities.

\section{MATERIALS AND METHODS}

\section{Subjects}

The feasibility study was performed as a prospective cohort study. Healthy young subjects $(n=20)$ from a mixed sample, composed of 8 males and 12 females (weight $50-90 \mathrm{~kg}$, height $155-195 \mathrm{~cm}$, aged $25-$ 50 years) participated in this study. The healthy young subjects were selected from the university student and staff population. Volunteer exclusion criteria included history of deformity of the spine, previous spinal surgery, back pain, pregnancy, and neurological disorder.

\section{The BalancAid and the Conventional Standing Method}

The proposed procedure employed the prototype of the BalancAid and the most recommended arm position. The BalancAid (Fig. 1) consists of a flat square board of $50.0 \mathrm{~cm} \times 50.0 \mathrm{~cm} \times 2.0 \mathrm{~cm}$ and a cylindrical disk with a diameter of $7 \mathrm{~cm}$ and a thickness of $2 \mathrm{~cm}$ placed in the center point of the bottom side. On the top side of the board, a guidance marker " $\mathrm{X}$ " and two lines were placed to guide a subject to place his feet at an angle of $45^{\circ}$ from the midline to make a neutral standardized stance (sub-talar joint neutral). A bar was placed on the board to force the feet to be placed in the center of the board. The idea of this system is that for each subject only one specific posture allows equilibrium of the board, and thus reproducibility is guaranteed.

Furthermore, the arms in the PA view while standing on the BalancAid were maintained straight in relaxed position on both sides without any supporting bars (Fig. 2a). The arm position in the LA view was the clavicular arm position with elbows flexed at $45^{\circ}$ and fists resting on the ipsilateral clavicles as described by Faro et al. ${ }^{9}$ (Fig. 2b).

The proposed BalancAid was compared with the so-called conventional standing on the ground method. It is the standing posture on the ground, which is usually applied in the hospital and suggested by the SRS. The feet were positioned in a sub-talar joint neutral position with each foot on the ground and standing in a relaxed manner. Each foot was placed with the dorsalis pedis on top of the appropriate " $\mathrm{X}$ " marked point on the floor. This procedure employed external lining supporting bars that enable the subject to put the hands to align the subject to the axis system. The PA view was obtained with both arms holding the supporting bars in the subject's left and right sides (Fig. 3a). In order to avoid any obscuring arm shadow in the LA view, the arms were positioned in such a way that the hands were able to hold a supporting bar (Fig. 3b).

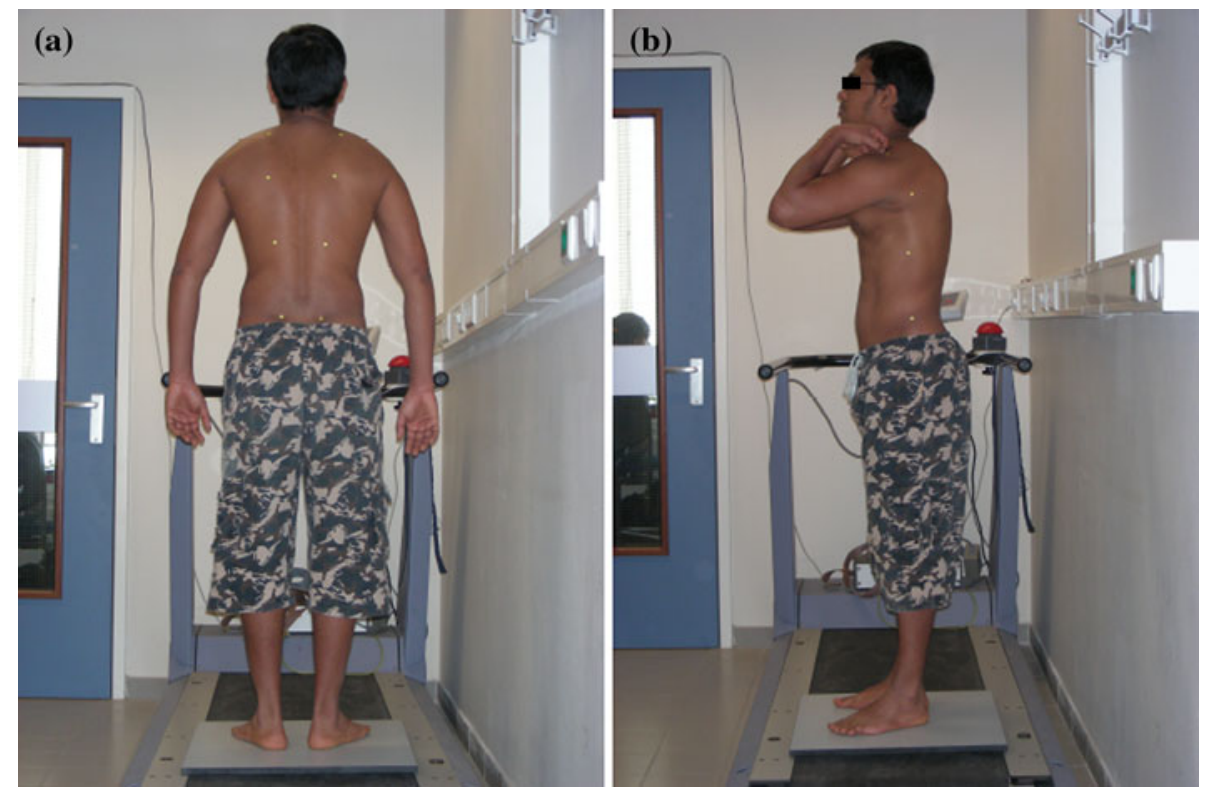

FIGURE 2. Arm positions in AP (a) and LA (b) view while standing on the BalancAid. 

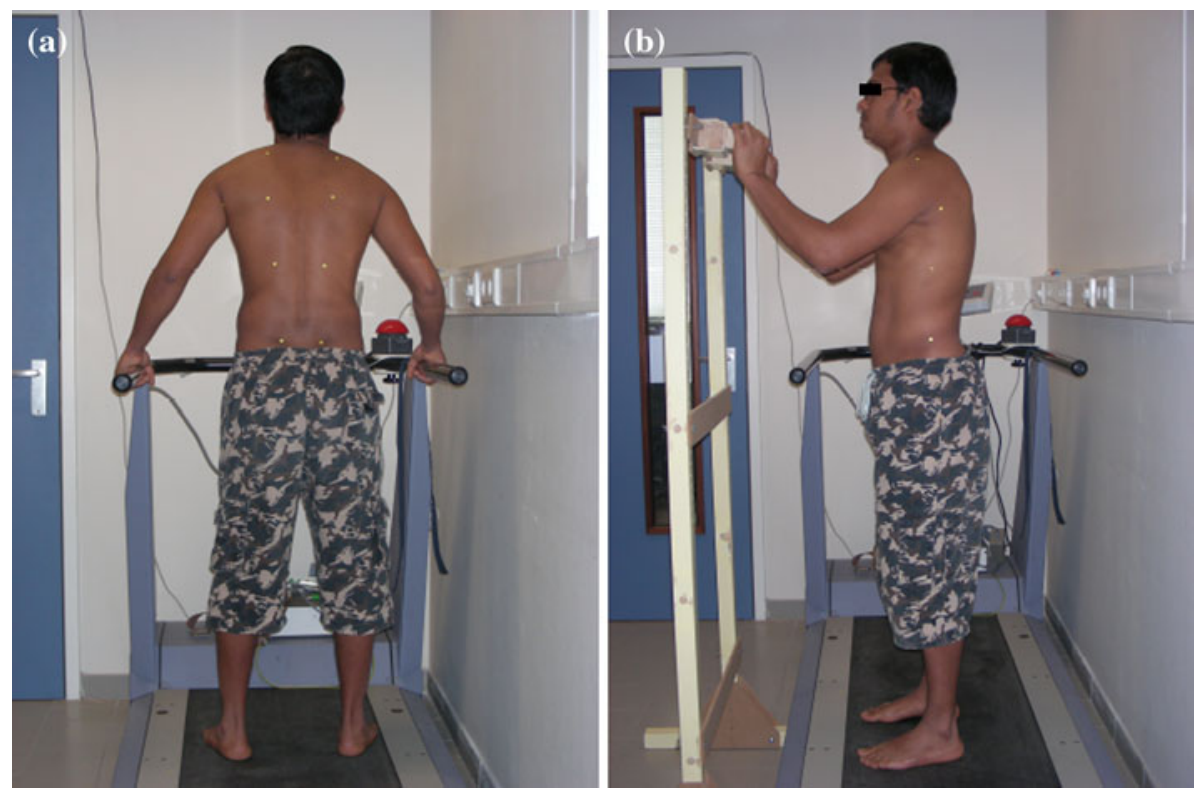

FIGURE 3. Arm positions in AP (a) and LA (b) view while standing in conventional method.

For both standing postures, the subject stood straight up. The head was positioned in a normal forward looking position with the eyes open. The chin was directed as if to avoid its shadow on the spinal $\mathrm{X}$-ray radiographs.

\section{Posture Quantification}

Body marks were applied for both PA and LA views (Fig. 4) and lines between body marks were drawn to quantify the body posture. The angles between the lines were utilized to define the position of the body.

(a)

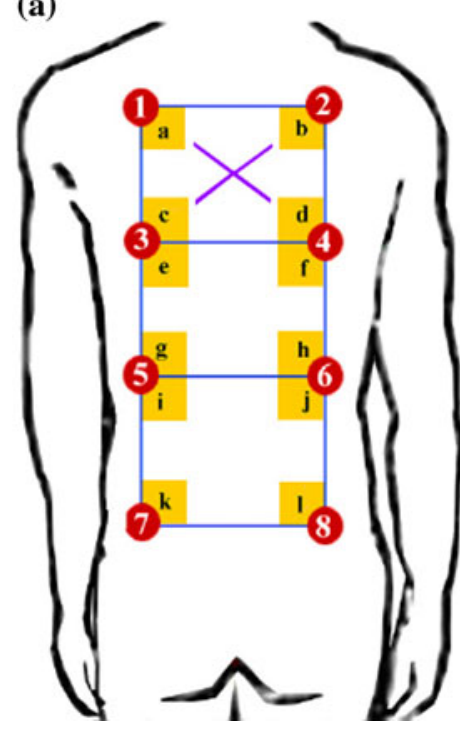

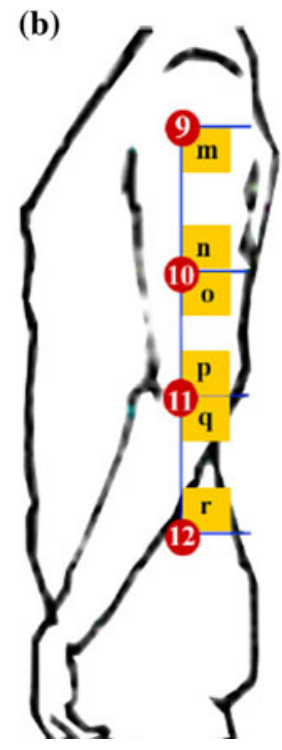

(b)

FIGURE 4. Body marks drawing in AP (a) and LA (b) view.

The subject needed to undress the torso to fully expose his/her back for body marks placement and examination. Eight markers on PA view and four markers on LA view were placed at sides of:

$-\mathrm{T} 3=$ root of the spine of the scapula (point 1 and 2 in PA view, and point 9 in LA view)

$-\mathrm{T} 7=$ inferior angle of the scapula (point 3 and 4 in PA view, and point 10 in LA view)

$-\mathrm{T} 12=$ posterior spinal connection of the last rib (point 5 and 6 in PA view, and point 11 in LA view)

- L4 $=$ highest point of the illiac crest (point 7 and 8 in PA view, and point 12 in LA view)

The four markers on LA view were placed in the same level as the markers on PA view. An extra calibration mark for calibration purposes was placed in the middle of body mark point 1,2, 3 and 4 .

The body marks were examined within two day measurements to investigate whether one day delay among the captures affected the posture reproducibility. Therefore, it is crucial to ensure that the body marks maintained in position on the body to prevent errors due to repositioning of the body marks. For that reason, the marking was applied to remain visible on the subject's body for the two consecutive day measurements.

\section{Measuring Set-up}

The measuring set-up of both procedures (standing on the BalancAid and on the ground) was conducted to mimic the set-up of the X-ray imaging of the spine 


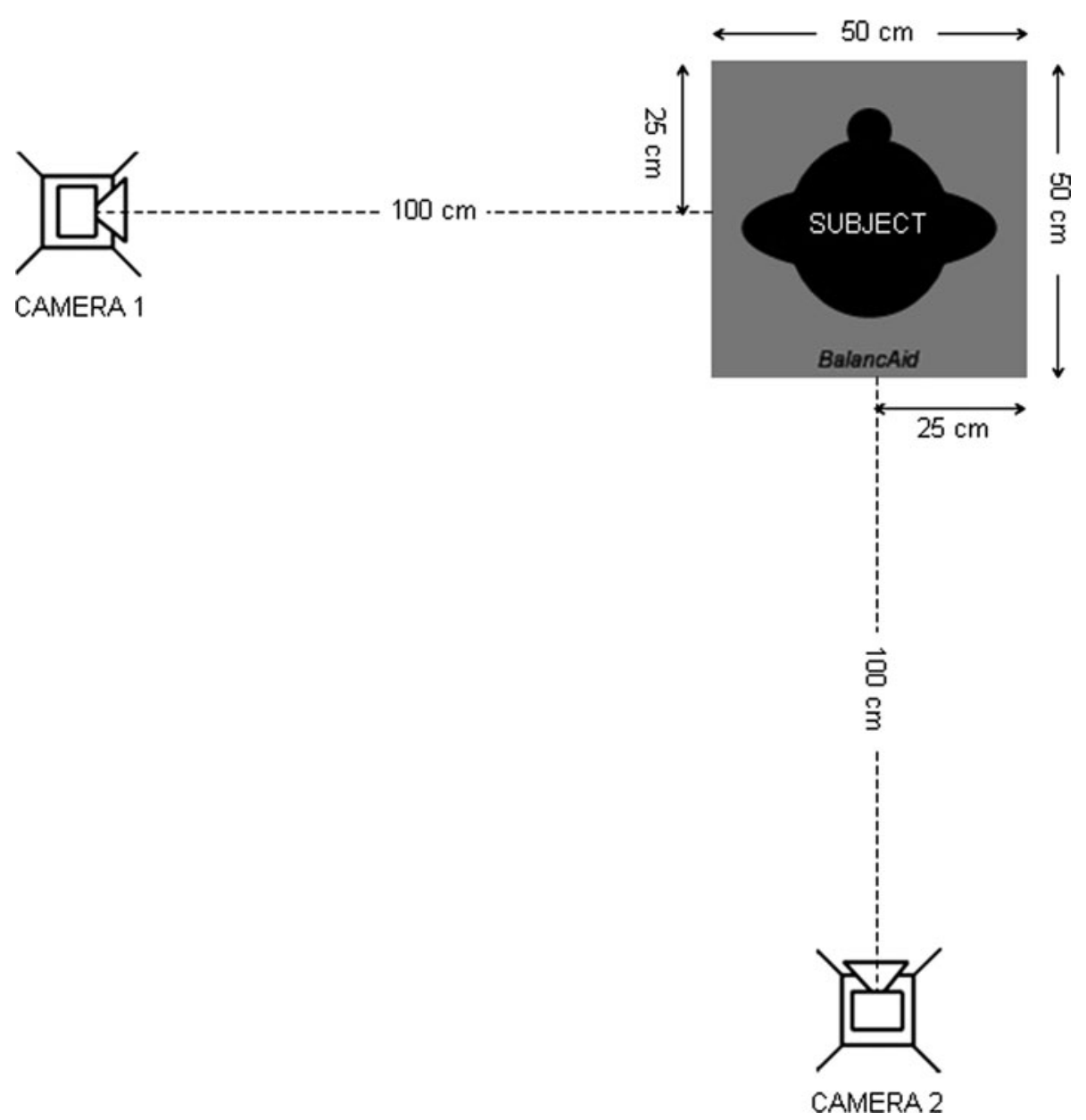

FIGURE 5. Photographic acquisition lay out of the posture reproducibility measurement with the subject standing on the BalancAid.

procedure. Two digital photo cameras were located perpendicularly each other at $1 \mathrm{~m}$ from the subject with the calibration mark in the center of the lens. The layout of the set-up, which consisted of three center points, is depicted in Fig. 5. Point $P$ indicates the position of the BalancAid device and of the subject.

\section{Protocol}

In order to compare between standing on the ground and on the BalancAid, each subject was examined with both methods in two consecutive days, after the information about the study was provided. Before the image acquisition, the cameras were adjusted on the proper setting, height, and distance. On each day, the subject was instructed to stand upright, first on the ground and then on the Balanc Aid. In order to determine the reproducibility, each subject repeated both procedures for 5 times and took a break in between. For the break relative to standing posture on the ground while walking around the examination room for approximately $5 \mathrm{~min}$. For the break relative to the BalancAid, the subject was asked to step on and off the BalancAid device with an interval of $5 \mathrm{~min}$. This session was repeated one day after by investigating the same body marks attached from the previous day. Seeing that diurnal variability influences the standing posture, ${ }^{1}$ the time of measurement on both days was made the same. All measurements were taken by one examiner.

\section{Data Analysis}

The body marks location was determined from the acquired photographs by taking the coordinates of the center of mass of each mark on both standing posture methods using Adobe Photoshop 7.0 (Adobe, San Jose, Calif) with the accuracy of 12 pixels $/ \mathrm{mm}$. Horizontal lines were drawn in the body marks on each PA point in the left side connected to its adjacent PA point on the right side of the back of the subject. Virtual vertical lines were drawn to connect PA points 
in each left and right side. Then, 12 PA angles (indicated by $a, b, c, \ldots, l)$ were defined between horizontal and vertical lines, see Fig. 4a. In LA, vertical lines were drawn to connect the LA points and a horizontal line was drawn through every LA point to determine the six LA angles (indicated by $m, n, o, \ldots, r$ ), see Fig. 4b. In order to avoid any redundancy in the analysis of the angles in PA views, in each quadrilateral, only three out of four angles were used for determining the posture. For the same reason, three out of the six angles in LA view were also omitted. In this regard, we choose angle $d$, $h, l, n, p$, and $r$ to be taken out from the analysis.

\section{Statistical Methods}

The most suitable method to evaluate the reproducibility between two repeated measurements was based on Lin's Concordance Correlation Coefficient (CCC) analysis that is referring to the Case-3A-model of McGraw and Wong ${ }^{20}$ and Chen and Barnhart. ${ }^{6}$ This reproducibility index confirmed the agreement between the two readings (from the same subjects) by assessing the expected squared deviance from the $45^{\circ}$ line through the origin (the concordance line). This method has the preferred characteristics for comparison of two repeated measurements and consistent estimation of sample counterparts, and asymptotic normality for bivariate normal data. ${ }^{16}$

We examined for each angle the intra subject reliability between sessions. The correlations were calculated out of all five trials per subject for each of two day measurements. The advantage was that it reported true reliability by considering any proportion of agreement that might have been arrived at due to chance. Statistical analysis was implemented using the programming language $\mathrm{R}$. The reproducibility of a method is considered to be high when the CCC value is close to maximum score of 1 .

In order to realize this method, we applied the following steps:

- A first mixed-effects model was estimated with two separated estimates of the standard deviation of both methods.

- A second mixed-effects model was estimated with one common estimate of the standard deviations of both methods.

- ANOVA test (with $p$-value) was utilized to examine whether there is a significant difference between the models.

- For each method and each angle, the CCC was calculated, and for each angle, the difference in CCC's was computed.

The bootstrap method on 1000 samples were performed to estimate the distribution of the difference in the CCC's. ${ }^{18}$ The $95 \%$ confidence interval was also employed to test for a significant difference.

The CCC analysis was performed for comparison between two repeated measurements; the conventional standing posture on the ground in two consecutive days $\left(\right.$ Ground $\left._{\text {day }} 1-2\right)$ and the proposed method using the BalancAid device in two consecutive days (Balanc Aid day 1-2).

\section{RESULTS}

Evaluation on all reproducibility results from healthy young subjects with good balance control as shown in Table 1 reveals that the CCC's of all angles were high for both standing postures. The high value of CCC's is in accordance with the high reproducibility. Seven out of nine angles $(a, c, e, f, g, i$, and $k)$ in PA view and two out of three angles $(m$ and $q)$ in LA view give higher CCC results on the BalancAid $_{\text {day } 1-2}$ method compared to the Ground day 1-2 method. The results from the ANOVA-test showed that angle $m$ in LA view has a significant difference in concordance between both methods in two-day measurements $\left(\mathrm{CCC}_{\text {Balanc Aid }}=0.904, \mathrm{CCC}_{\text {Ground }}=0.710, p>0.005\right)$. This is an indication that the Balanc Aid day 1-2 method in this angle is considerably more reproducible than the Ground day 1-2 method. Although the other angles showed no significant difference $(p>0.005)$ between the Ground day 1-2 $_{1-2}$ and the BalancAid day 1-2 method, the CCC of most other angles revealed that the BalancAid method still improves the reproducibility.

TABLE 1. Lin's Concordance Correlation Coefficient (CCC) of 12 angles for both measurement methods, ground and BalancAid, resulted that angle $a, c, e, f, g, i$, and $k$ in PA view and angle $m$ and $q$ in LA vier give higher reproducibility.

\begin{tabular}{lccc}
\hline Angle & $\begin{array}{c}\text { CCC } \\
\text { ground }\end{array}$ & $\begin{array}{c}\text { CCC } \\
\text { BalancAid }\end{array}$ & $\begin{array}{c}p \text {-Value based } \\
\text { on the comparison of the } \\
\text { two mixed effects models }\end{array}$ \\
\hline PAa & 0.884 & 0.895 & 0.4744 \\
PAb & 0.909 & 0.895 & 0.0528 \\
PAc & 0.898 & 0.909 & 0.4133 \\
PAe & 0.907 & 0.922 & 0.2089 \\
PAf & 0.946 & 0.952 & 0.3369 \\
PAg & 0.931 & 0.944 & 0.0877 \\
PAi & 0.847 & 0.874 & 0.0893 \\
PAj & 0.889 & 0.878 & 0.1715 \\
PAk & 0.927 & 0.937 & 0.1913 \\
LAm & 0.710 & 0.904 & 0.0001 \\
LAo & 0.761 & 0.715 & 0.4973 \\
LAq & 0.750 & 0.830 & 0.0675 \\
\hline
\end{tabular}

Angle $m$ has a significant difference in concordance between both methods in two-day measurements. 


\section{DISCUSSION}

An irreproducible posture could lead to a false measurement, a wrong diagnosis or an unnecessary or delayed treatment. In the conventional X-ray radiography procedure, a radiographer usually instructs the scoliotic patient to stand up in a relaxed position, as it is known that the relaxed position is the functional balance posture. However, many relaxed positions are possible, and thus inconsistent postures may be taken. Therefore, it is difficult to ensure a reproducible state in this posture. The recommendation of the $\mathrm{SRS}^{29}$ on the use of devices to standardize the posture during taking X-rays has been widely implemented and proven to improve the reproducibility of the posture.

Bernau $^{4}$ employed the body weight distribution through both feet and visual direction while the subject was standing on a pair of weight scales to obtain the balance control. This study shows that the reproducible posture can be obtained by taking a balanced posture in an equally distributed body weight. Furthermore, focusing the eyes in a certain direction is possibly to correct the posture reproducibility. Another study performed by Koreska et al. ${ }^{14}$ implemented the "Throne" to reproduce the positioning of the patients. However, this device merely enabled the patient to be imaged in sitting position, while the standardized method for curvature measurement out of X-rays imposes a standing position. Furthermore, this method still did not assure the balance in the sagittal plane. One more attempt which included the standing posture and balance control was a balancing plate made by Kohlmaier et al. ${ }^{13}$ Although this balance-like positioning device employed the balance control of the body, this device merely considered the balance in the frontal plane which was only possible for a specific upright position; thus, balance in the sagittal plane and the arm positioning were not prescribed.

On the basis of this problem, the BalancAid is proposed in this study to attain a balanced reproducible posture in all planes by referring to the see-saw principle in finding the stability. We compared the posture reproducibility between the two standing methods, the Ground $_{\text {day 1-2 }}$ and the BalancAid day 1-2, and evaluated the performance of the BalancAid in realizing posture reproducibility during X-ray radiography on healthy young subjects with good balance control. The body marks appeared to remain in place within the two consecutive measurement days and thus did not introduce an error.

The reproducibility of the standing posture was represented by the concordance of the repeated measurement of both methods. The higher concordance results of nine out of 12 cases $(a, c, e, f, g, i, k, m$, and q) confirm a better reproducibility of the Balanc$\operatorname{Aid}_{\text {day 1-2 }}$ in relation to the Ground day 1-2 method. This is also fortified by the significant different result obtained in angle $m$. Even though only one angle resulted in a significant difference, the CCC calculation presents good concordance $(>0.7)$ for all the angles. The results from the healthy young subjects may signify that the posture of the subjects is highly reproducible on repeated measurements between two day measurements on the BalancAid.

Our posture reproducibility experiment was performed with healthy young subjects with good balance control. Therefore, the current results are especially valid for that group. The healthy volunteers participating in this feasibility study may also represent other patients who need X-Rays for other diagnostic purposes and that will benefit from a reproducible posture, especially between AP/PA and LA position. Further study will be performed on young scoliotic patients to find out whether the BalancAid is also beneficial for young patients with moderate or severe scoliosis who need to take a spinal X-ray radiographs to follow the progression over time.

We also found that the concordances of the PA angles are on average higher than the LA angles. This may be an indication that the reproducibility in the LA position is more difficult to realize than in the PA position. In addition, the application of the arm positioning in the LA view which is known to affect the reproducibility may be associated with our finding of lower reproducibility in the LA angles. Previous armpositioning studies ${ }^{1,9,11,19}$ have been focused on the effect of the arm positioning on the reliability of the spinal deformity measurement. These studies have provided evidence that positioning the arm in such a way that it minimizes the imbalanced effect in LA view is capable of enhancing the sagittal spinal alignment and proposed several arm positionings to apply. Furthermore, comparison among arm positions ${ }^{10,19,30}$ proposed in these studies have considered the fist on clavicle arm position to be the best choice to represent a functional balance while still allowing an adequate LA view of the spine in the X-ray radiography. This arm position is chosen for the reason that it has the closest sagittal vertical axis to the functional standing posture. Therefore, we applied the fist on clavicle arm position in the posture reproducibility measurement using the BalancAid. However, thus far, no standardized procedure could be provided for the scoliosis patients due to individuality of every deformity. Our experiences with the arm position are that the method used with the BalancAid could be applied for all subjects without any problems. 
A limitation of this study is that there are sources of variability which we have not examined. Defining the center of the body marks was done manually from the body marks picture. This could be one cause of measurement variability. Also the deviations from the standardized camera setting and layout may influence the measurement accuracy. However, since we derived that these errors are small, no significant influence was obtained.

Another limitation concerns about the posture of the patients. In actual fact, generating a reproducible relaxed standing position is impossible even if it is a functional standing posture which represents the actual spinal deformity. The BalancAid, which forces the posture to stand in a specific upright position, still cannot produce the real relaxed position. The position during standing on the BalancAid is indeed not as natural as the relaxed standing one; however, the balancing effect brings about the standing position where the center of gravity is always located in the same position. Therefore, the reproducibility is guaranteed. Furthermore, we have proven that this posture generates better reproducibility resulting in a more truthful analysis of the spinal deformity progression over time.

Thus far, no comparison to other previous studies $^{4,13,14}$ on posture reproducibility devices could be made due to differences in imposing the standing posture and the arm positioning. Therefore, a comparison to the conventional standing method which is generally applied in the hospital was considered to be adequate to represent how far the BalancAid solves the posture reproducibility problem.

\section{CONCLUSION}

The posture reproducibility measurement on healthy young subjects by the Balanc Aid in general yields higher reproducibility than standing on the ground by grasping a supporting bar. A significant difference in one angle also confirms that standing on the BalancAid in such angle is more reproducible than standing on the ground. An investigation on a single arm position for the AP/PA and LA views without changing the position may be needed to avoid ambiguity with the reproducibility.

\section{ACKNOWLEDGMENTS}

This research is made possible from the financial supports of the Schlumberger Foundation-Faculty for the Future and Bernoulli Foundation-University of Groningen.

\section{OPEN ACCESS}

This article is distributed under the terms of the Creative Commons Attribution Noncommercial License which permits any noncommercial use, distribution, and reproduction in any medium, provided the original author(s) and source are credited.

\section{REFERENCES}

${ }^{1}$ Aota, Y., T. Saito, M. Uesugi, K. Ishida, K. Shinoda, and K. Mizuma. Does the fists-on-clavicles position represent a functional standing position? Spine 34:808-812, 2009.

${ }^{2}$ Beauchamp, M., H. Labelle, G. Grimard, C. Stanciu, B. Poitras, and J. Dansereau. Diurnal variation of Cobb angle measurement in adolescent idiopathic scoliosis. Spine 18:1581-1583, 1993.

${ }^{3}$ Beaulieu, M., C. Toulotte, L. Gatto, C. H. Rivard, N. Teasdale, M. Simoneau, and P. Allard. Postural imbalance in non-treated adolescent idiopathic scoliosis at different periods of progression. Eur. Spine J. 18:38-44, 2009.

${ }^{4}$ Bernau, A. Zur frage der reproduzierbarkeit von rönthenaufnahmen der wirbelsäule im stehen (On the problem of the reproducibility of X-rays of the spinal column standing). Z. Orthop. 122:150-151, 1984.

${ }^{5}$ Capasso, G., N. Maffulli, and V. Testa. The validity and reliability of measurements in spinal deformities: a critical appraisal. Acta Orthop. Belg. 58:126-135, 1992.

${ }^{6}$ Chen, C. C., and H. X. Barnhart. Comparison of ICC and $\mathrm{CCC}$ for assessing agreement for data without and with replications. Comput. Stat. Data Anal. 53:554-564, 2008.

${ }^{7}$ Cobb, J. R. Outline for the study of scoliosis. AAOS Instr. Course Lect. 5:261-275, 1948.

${ }^{8}$ DeSmet, A. A., J. E. Goin, M. A. Asher, and H. G. Scheuch. A clinical study of the differences between the scoliotic angles measured on posteroanterior and anteroposterior radiographs. J. Bone Joint Surg. 64:489-493, 1982.

${ }^{9}$ Faro, F. D., M. C. Marks, J. Pawelek, and P. O. Newton. Evaluation of a functional position for lateral radiograph acquisition in adolescent idiopathic scoliosis. Spine 29: 2284-2289, 2004.

${ }^{10}$ Hayashi, K., V. V. Upasani, J. B. Pawelek, C. E. Aubin, H. Labelle, L. G. Lenke, R. Jackson, and P. O. Newton. Three-dimensional analysis of thoracic apical sagittal alignment in adolescent idiopathic scoliosis. Spine 34:792797, 2009.

${ }^{11}$ Horton, W. C., C. W. Brown, K. H. Bridwell, S. D. Glassman, S. I. Suk, and C. W. Cha. Is there an optimal patient stance for obtaining a lateral 36" radiograph? A critical comparison of three techniques. Spine 30:427-433, 2005.

${ }^{12}$ Keim, H. A. Scoliosis. Clin. Symp. 30:1-30, 1978.

${ }^{13}$ Kohlmaier, W., K. Lercher, and C. Tschauner. Einsatz einer dynamischen wippe zur standardisierten aufnahmetechnik von röntgengesamtaufnahmen der wirbelsäule im stehen bei kindern (Balance-like positioning device for taking standardized X-rays of the spine when the patient is standing). Radiologe 35:60-66, 1995.

${ }^{14}$ Koreska, J., E. P. Schwentker, A. M. Albisser, D. A. Gibson, and R. H. Mills. A simple approach to standardized spinal radiographs. Med. Instrum. 12:59-63, 1978. 
${ }^{15}$ Le Blanc, R., H. Labelle, B. Poitras, C. H. Rivard, and J. Kratzenberg. 3-D evaluation of posture in normal and scoliotic adolescents. Ann. Chir. 50:631-636, 1996.

${ }^{16}$ Lin, L. I. A concordance correlation coefficient to evaluate reproducibility. Biometrics 45:255-268, 1989.

${ }^{17}$ Loebl, W. Y. Measurement of spinal posture and range of spinal movement. Rheumatology 9:103-110, 1967.

${ }^{18}$ Manly, B. F. Randomization, Bootstrap, and Monte Carlo Methods in Biology. Chapman and Hall, 1997.

${ }^{19}$ Marks, M., C. Stanford, and P. Newton. Which lateral radiographic positioning technique provides the most reliable and functional representation of a patient's sagittal balance? Spine 34:949-954, 2009.

${ }^{20} \mathrm{McGraw}, \mathrm{K}$. O., and S. P. Wong. Forming inferences about some intraclass correlation coefficients. Psychol. Methods 1:30-46, 1996.

${ }^{21}$ Mirovsky, Y., A. Blankstein, and N. Shlamkovitch. Postural control in patients with severe idiopathic scoliosis: a prospective study. J. Pediatr. Orthoped. B 15:168-171, 2006.

${ }^{22}$ Morin, D. M., J. E. Lonstein, M. Stovall, D. G. Hacker, N. Luckyanov, and C. E. Land. Breast cancer mortality after diagnostic radiography: findings from the US Scoliosis Cohort Study. Spine 25:2052-2063, 2000.

${ }^{23}$ Nault, M. L., P. Allard, S. Hinse, R. Le Blanc, O. Caron, H. Labelle, and H. Sadeghi. Relations between standing stability and body posture parameters in adolescent idiopathic scoliosis. Spine 27:1911-1917, 2002.

${ }^{24}$ O'Beirne, J., C. Goldberg, F. E. Dowling, and E. E. Fogarty. Equilibrial dysfunction in scoliosis-cause or effect? J. Spinal Disord. Tech. 2:184-189, 1989.

${ }^{25}$ Oda, M., S. Rauh, P. B. Gregory, F. N. Silverman, and E. E. Bleck. The significance of roentgenographic measurement in scoliosis. J. Pediatr. Orthop. 2:378-382, 1982.

${ }^{26}$ Pruijs, J. E. H., M. Hageman, W. Keessen, R. Meer, and J. C. Wieringen. Variation in Cobb angle measurements in scoliosis. Skeletal Radiol. 23:517-520, 1994.

${ }^{27}$ Schmidt, J., and F. Gassel. Clinical use of the simple 3Dcalculation in scoliosis. Skeletal Radiol. 23:43-48, 1994.

${ }^{28}$ Schumpe, G., P. Hofmann, and H. Rössler. Problematik der verlaufskontrolle bei skoliosepatienten (Follow-up problems in scoliosis patients). Z. Orthop. 122:243-254, 1984.

${ }^{29}$ Stokes, I. A. F. Three-dimensional terminology of spinal deformity: a report presented to the Scoliosis Research Society by the Scoliosis Research Society Working Group on 3-D terminology of spinal deformity. Spine 19:236-248, 1994.

${ }^{30}$ Vialle, R., N. Levassor, L. Rillardon, A. Templier, W. Skalli, and P. Guigui. Radiographic analysis of the sagittal alignment and balance of the spine in asymptomatic subjects. J. Bone Joint Surg. 87:260-267, 2005. 